\title{
Pictorial enhancement of text memory: Limitations imposed by picture type and comprehension skill
}

\author{
PAULA J. WADDILL and MARK A. McDANIEL \\ Purdue University, West Lafayette, Indiana
}

\begin{abstract}
We examined the kinds of information in a prose passage that is better remembered when depictive illustrations are embedded in the passage than when the passage contains no illustrations. Experiment 1 showed that (1) pictures depicting details effectively increased recall of those details and (2) pictures depicting relationships effectively increased recall of that relational information (relative to a no-picture control condition). In Experiment 2, comprehension skill was found to modulate the general effects obtained in Experiment 1. Detail pictures enhanced the recall of targeted details for all skill levels. Relational pictures enhanced recall of pictured relational information for highly skilled and moderately skilled comprehenders, but not for less skilled comprehenders. Because there were no recall differences across the different skill levels in the nopicture control condition, it is suggested that pictures may serve to enable processing in which readers would not necessarily engage under ordinary circumstances. Pictures, however, did not appear to compensate for limitations reflected in lower scores on a standardized test of reading comprehension.
\end{abstract}

The role of illustrations in promoting text memory has been of interest to researchers for some time. An approach that has proven fruitful in such endeavors is one that classifies text illustrations in terms of various functions that they may perform (Levin, 1981; Levin, Anglin, \& Carney, 1987; Mayer \& Gallini, 1990). Pictures may serve to decorate the passage, to represent information in the text, to transform the information into a more memorable form, to help the reader organize the text, or to help the reader understand and interpret the text.

Classifying the various functions performed by illustrations is a useful framework from which to begin a systematic study of picture effects, and we have used such a framework as a starting point. We have extended it, however, by considering not only general functional categories of pictures but also the different kinds of text information toward which pictures may direct processing. Previous work in text processing and memory has indi-

This research was presented in part at the annual meeting of the Midwestern Psychological Association, Chicago, May 1991, and at the Workshop on Comprehension of Graphics, Tubingen, Germany, October 1991 (the workshop paper will appear in a forthcoming publication of the Workshop proceedings). This work was supported in part by the National Institute of Child Health and Human Development Grant HD23984 awarded to the last author and in part by the Purdue Research Foundation Grant 69014343 awarded to both authors. We thank Gilles Einstein for his input on the materials and for his helpful suggestions on other aspects of this project. We also thank Margaret Jean Intons-Peterson and three anonymous reviewers for their helpful comments on an earlier version of this article. Correspondence concerning this article should be addressed to P. Waddill, who is now at Psychology Department, University of Scranton, Scranton, PA 18510-4596. cated that good recall depends upon the processing of at least two kinds of information: proposition-specific information and relational information (Einstein, McDaniel, Bowers, \& Stevens, 1984; Einstein, McDaniel, Owen, \& Cote, 1990; McDaniel, Einstein, Dunay, \& Cobb, 1986). Proposition-specific information is information that instantiates or describes an individual concept or idea unit in studied material. One category of proposition-specific information that is particularly relevant to text is that of details. In a text about avalanches in the Himalayas, for example, the statement, "The glaciers have walls of ice six hundred to one thousand feet wide," includes detail information about the specific size of the walls (600 to $1,000 \mathrm{ft}$ ). Relational information, on the other hand, represents the integration or organization of several concepts or idea units. In texts, relational information includes causal relations that connect ideas in terms of cause and effect (e.g., "The plasticity of the ice allows Kanchenjunga to develop enormously thick hanging glaciers with walls of ice six hundred to one thousand feet wide") and contrastive information that compares or contrasts different concepts or sets of concepts from the text (e.g., "Avalanches are more frequent in the Alps, but none approach the magnitude of the Kanchenjunga avalanches").

In previous work (Waddill, McDaniel, \& Einstein, 1988), we found that different kinds of pictures could indeed enhance the recall of different kinds of text information. Illustrations depicting relational information enhanced the recall of the pictured relational information in a fairy tale, and illustrations depicting detail information enhanced the recall of the pictured detail information in an expository text. Detail pictures did not enhance recall 
of details in the fairy tale, nor did relational pictures enhance the recall of relational information in the expository passage. In our present research, we were curious to see if this pattern of enhancement held generally or if it was possible to modulate it by altering characteristics of the illustrations themselves. The present experiments were further motivated by Haring and Fry's (1979) finding that pictures that included both relational and detail information enhanced recall of relational but not detail information in a narrative. Haring and Fry concluded that illustrations might not be beneficial in aiding recall of detail information, a conclusion that contradicted our previous results with expository texts.

In the present research, we sought to determine conditions under which pictures might enhance the recall of detail and relational information. To more carefully and systematically investigate this issue, we focused on expository text and gave careful consideration to the way in which the illustrations depicted the different types of information. Haring and Fry included both detail and relational information in their pictures. This mixing of information type might have contributed to their failure to find beneficial effects of pictures for recall of details. In our experiments, therefore, we used illustrations designed to target primarily one kind of information (either detail or relational).

Furthermore, an examination of the relational pictures used in Waddill et al. (1988) suggested that our relational illustrations might not have depicted conceptual relationships as clearly as we had intended. One illustration, for example, targeted causal and contrastive information about Himalayan glaciers being tougher and less brittle than Alpine glaciers because the two regions had different day and night temperature ranges. The picture consisted of two frames. The left frame, labeled Alps, showed a glacier with sharp edges. The right frame, labeled Kanchenjunga, showed a glacier with rounded edges covered with snow. It is possible that readers might have processed this illustration as two detail pictures rather than as a twoframe comparative, relational picture.

Some support for this supposition comes from the results of Waddill et al's (1988) second experiment. In that experiment, subjects reading the expository passage were specifically told to pay attention to relationships among ideas in the text. Subjects in the relational picture group showed a small increase in recall of targeted relational information (relative to a no-picture control group), but this difference was not statistically reliable. Thus, even with instructions to attend to relational information and to use the pictures to help them to do so, the relational pictures did not aid recall of targeted information. These data left open the question of whether or not illustrations could ever enhance recall of relational information in expository text. In the present study, therefore, we redrew three of the original relational pictures and substituted a new fourth one. We attempted to draw all of these pictures so that they more clearly depicted the causal and contrastive relationships in which we were interested. We also redrew three of our original detail illustrations and substituted four new ones.

We first report an experiment that demonstrates that relational pictures can, in fact, be designed in such a way as to enhance the recall of relational information from expository text. Experiment 1 also replicates our original (Waddill et al., 1988) finding that detail pictures can enhance recall of targeted detail information.

Experiment 2 had two purposes. First, we sought to demonstrate via an additional experiment the reliability of the overall general pattern in Experiment 1. Second, there is good reason to believe that individual ability also plays a role in determining the mnemonic effectiveness of text illustrations (see, e.g., Gernsbacher, Varner, \& Faust, 1990; Levin, 1973; Mayer \& Gallini, 1990). In Experiment 2, therefore, we considered the question of whether or not individual differences in reading ability modulate the general pattern of picture effects. Prior to Experiment 2, we outline differing viewpoints of how reading ability might influence the effectiveness of illustrations. We then test the predictions generated by those viewpoints.

\section{EXPERIMENT 1}

\section{Method}

Subjects. The subjects were 48 Purdue University undergraduates who participated in partial fulfillment of an introductory psychology course requirement. The subjects were tested in groups of 1 to 4 persons each.

Materials. The passage was based on the expository text used by Waddill et al. (1988) entitled, "Kanchenjunga, A Very Dangerous Mountain Range." We modified the text slightly by adding more specific details. We also improved the conceptual coherence of the text by rewriting it in such a way that each sentence (except the first) contained a repetition of a concept or idea unit from the immediately preceding sentence. This rewritten version contained 17 sentences and 61 idea units (where idea units were general approximations of the propositions from Kintsch's, 1974, method of analyzing text meaning)

Three versions of the passage were constructed. In the detailpicture condition, the passage was accompanied by seven blackand-white line drawings. Three of these drawings were redrawn versions of the original detail pictures in Waddill et al. (1988) and three were new. Each drawing was designed primarily to target one detail conveyed by one idea unit in the story. Take, for example, the detail information in the sentence, "The glaciers have walls of ice that are 600 to 1,000 feet wide." Figure 1 shows the accompanying illustration, designed to target the size information. Each detail picture was placed on the same page as the sentence containing the illustrated detail. Unillustrated sentences were grouped together on separate pages. The pages were compiled into an 11-page booklet.

In the relational-picture condition, the passage was accompanied by four line drawings. Three of these were redrawn versions of the original (Waddill et al., 1988) pictures and one was new. Each drawing targeted causal or contrastive relationships among four or five idea units in the story. The following two sentences contain an example of relational information relative to differences between the Alps and the Himalayas: "The ice in the Alpine district breaks away much more often than the ice in the Kanchenjunga district. Thus, even though the Alps have more frequent avalanches than the Himalayas, none approach the magnitude of the Kanchenjunga 


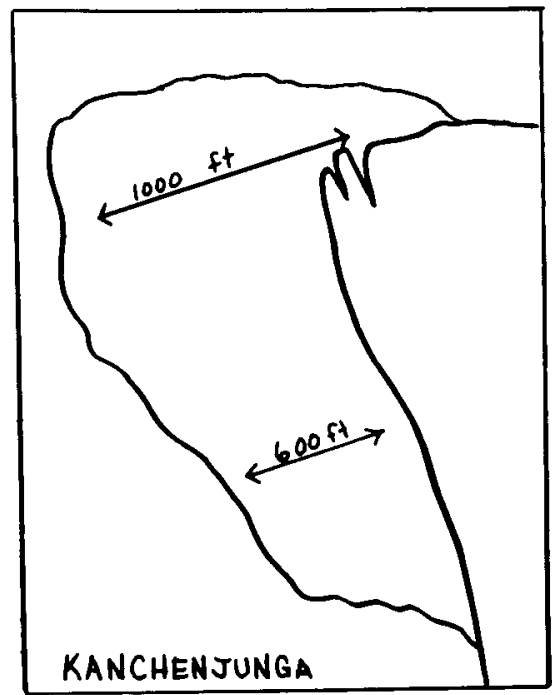

Figure 1. Example of a detail picture used in Experiments 1 and 2 to target size information. avalanches." Figure 2 shows the accompanying illustration designed to target the contrasts between Himalayan and Alpine avalanches. Each relational picture was placed on the same page as the sentence or sentences containing the illustrated idea units. Unillustrated sentences were grouped together on separate pages. The relationalpicture booklet contained seven pages. Booklets in the no-picture control condition were constructed in the same fashion as the booklets for the picture conditions. Half of the control booklets had pages in which the sentences were grouped as in the detail-picture booklets, and half had pages in which the sentences were grouped as in the relational-picture booklets.

Design and Procedure. Picture condition was manipulated between subjects. Fifteen subjects were randomly assigned to the no-picture control condition, 16 subjects to the detail-picture condition, and 17 subjects to the relational-picture condition.

An incidental learning situation was used. The subjects were told that the purpose of the study was to investigate comprehension and that they would later be asked to rate how easy the story was to understand. No mention was made of a subsequent memory task. Subjects in the picture conditions were told to pay attention to the pictures in order to aid their comprehension. When the subjects finished reading the passage, their reading times were recorded. The booklets were removed, and they were asked to rate their comprehension on a scale ranging from 1 (didn't comprehend at all) to 5 (comprehended very well). After completing the rating task,
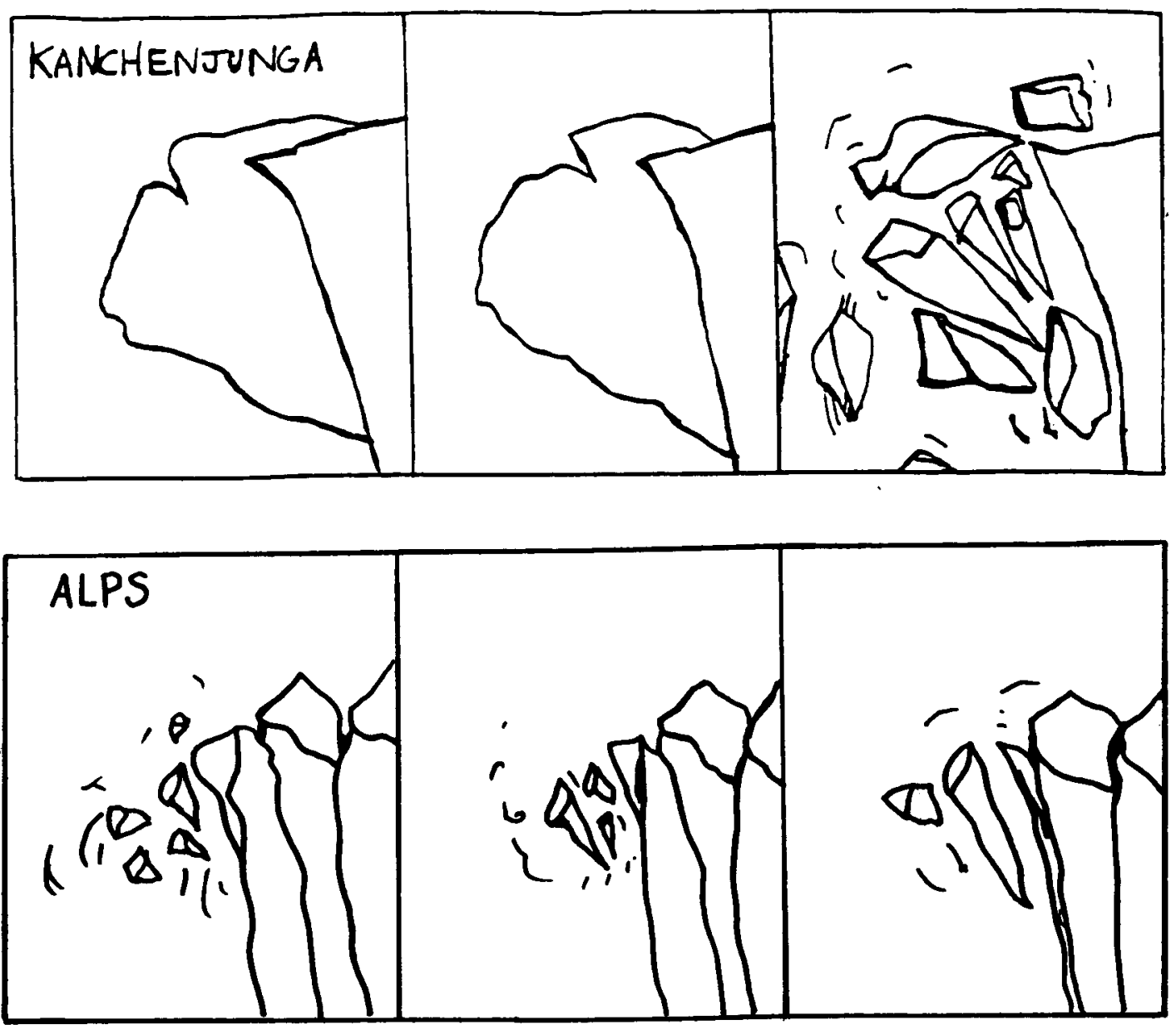

Figure 2. Example of a relational picture used in Experiments 1 and 2 to target contrastive information. 
they solved math problems for $5 \mathrm{~min}$. Next, they were asked to write down as much of the passage as they could remember and were given as much time as they needed to do so. When they had completed the free-recall task, they were asked to rate their degree of prior knowledge of the information discussed in the passage using a scale ranging from 1 (none of it) to 7 (all of it). Finally, the subjects received a postexperimental questionnaire asking them if they had expected a memory test. Nineteen subjects indicated that they had expected and prepared for a memory test. These subjects were replaced with 19 subjects who were from the same subject pool and who did not expect or prepare for a memory test.

\section{Results}

Comprehension. The rejection level for all analyses was set at .05 . Table 1 contains the means for reading time, comprehension rating, and rating of prior knowledge. Self-ratings of prior knowledge were uniformly low, and there were no significant differences among the groups (all $F \mathrm{~s}<1$ ). Comprehension ratings also did not differ significantly among the groups (all $F \mathrm{~s}<1$ ). The groups did differ significantly in time to read the passage $\left[F(2,45)=4.45, M S_{\mathrm{e}}=.29\right]$. A Newman-Keuls analysis indicated that detail and relational pictures significantly increased reading time, relative to the no-picture control condition. Reading times for the two picture conditions were not significantly different from each other.

Free recall. Two scorers scored a random subset of 10 protocols (4 protocols from the control group and 3 each from the two picture groups). The scorers were blind to the condition from which each protocol was drawn. Following Feldman (1985), each idea unit recalled was given a rating of 2 (entirely correct), 1 (partially correct), or $\mathbf{0}$ (incorrect). An entirely correct idea unit veridically conveyed the gist of the target idea unit. A partially correct idea unit did not precisely capture the target idea unit, but closely related to it. Because the correlation between the two raters was .98 , the remaining protocols were scored by one of the scorers.

Table 2 presents the proportions of the total possible scores for the idea units that were depicted in the detail and relational pictures (target propositions) and for the idea units that were not depicted in the illustrations (nontarget propositions). Paralleling Waddill et al. (1988), planned comparisons were used to evaluate the effects of each type of illustration on the recall of a given type of information. These comparisons consisted of tests of the

Table 1

Experiment 1: Average Reading Times, Comprehension Ratings, and Prior Knowledge Ratings as a Function of Picture Condition

\begin{tabular}{lccc}
\hline & \multicolumn{3}{c}{ Picture Condition } \\
\cline { 2 - 4 } \multicolumn{1}{c}{ Dependent Measure } & Control & $\begin{array}{c}\text { Detail } \\
\text { Pictures }\end{array}$ & $\begin{array}{c}\text { Relational } \\
\text { Pictures }\end{array}$ \\
\hline Reading time & 2.36 & 2.87 & 2.85 \\
Comprehension rating* & 4.07 & 4.29 & 4.19 \\
Prior knowledge rating $\dagger$ & 1.20 & 1.59 & 1.88 \\
\hline Note-Reading time is given in minutes. & * Larger numbers indicate \\
higher comprehension rating (highest possible $=5$ ). † †arger num- \\
bers indicate higher prior knowledge rating (highest possible $=7$ ).
\end{tabular}

Table 2

Experiment 1: Mean Proportion of Total Possible Score for Free Recall of Target Detail, Target Relational, and Nontarget Information as a Function of Picture Condition

\begin{tabular}{lllll} 
& \multicolumn{3}{c}{ Picture Condition } \\
\cline { 3 - 5 } Information Type & & Control & $\begin{array}{c}\text { Detail } \\
\text { Pictures }\end{array}$ & $\begin{array}{c}\text { Relational } \\
\text { Pictures }\end{array}$ \\
\cline { 1 - 4 } Target Detail & & $.29(.20)$ & $.45(.40)$ & $.28(.21)$ \\
Target Relational & $.15(.17)$ & $.15(.15)$ & $.23(.24)$ \\
Nontarget & .14 & .14 & .17 \\
\hline
\end{tabular}

Note-Scores reflect the proportions of total possible scores for target detail, target relational, and nontarget information $(24,36$, and 62 , respectively). Scores in parentheses represent total scores after elimination of problematic relational picture from analysis (see text for explanation).

control group against each of the picture groups for each kind of information (target detail, target relational, nontarget). Error terms used in the comparisons were those taken from separate analyses for recall of each kind of information (target detail, target relational, nontarget).

The recall of detail information was significantly greater in the detail picture condition than in the control condition $\left[F(1,30)=10.20, M S_{\mathrm{e}}=.02\right]$. Recall of detail information was not, however, enhanced by relational pictures $(F<1)$. Recall of relational information was significantly enhanced by relational pictures $\left[F(1,29)=4.95, M S_{\mathrm{e}}=\right.$ $.01]$, but not by detail pictures $(F<1)$. Pictures did not significantly enhance the recall of nontarget information (all $F \mathrm{~s}<1$ ).

\section{Discussion}

In this experiment, as in the previous work by Waddill et al. (1988), pictures did not significantly enhance the recall of information not targeted by the picture. For pictured information, the present results supported Waddill et al.'s earlier findings for detail pictures embedded in an expository passage. Recall of target detail information was enhanced by the presence of illustrations depicting those target details. Even more importantly, the present experiment demonstrated that, unlike Waddill et al., recall of targeted relational information in an expository passage was enhanced by the inclusion of relational illustrations. Waddill et al.'s failure to find such enhancement may have been due to their using ineffective relational pictures, or perhaps it was due to "relational" and "detail" not being well-defined categories where pictures are concerned. ${ }^{1}$ To address these concerns, we conducted a picture-text information matching task (concurrent with Experiment 2). We sought to determine whether or not subjects could accurately discriminate the differential emphasis on information in detail and relational pictures. To provide a conservative estimate of the veracity with which the pictures conveyed the intended level of information, the subjects were required to process the pictures when not explicitly matched with particular segments of text.

Twenty-three subjects who did not participate in Experiment 2 were selected from the same subject pool. One group of 11 subjects (the new picture group) first read 
the unillustrated version of the Kanchenjunga passage used in Experiment 1 . After reading the story, they were given a booklet containing a list of all idea units in that story and a separate booklet containing the seven detail and four relational pictures. Each picture was placed on a separate page without any accompanying text, and the pages were placed in random order. Participants were instructed to write down for each picture the idea units they thought the picture illustrated. A second group of 12 subjects (the original picture group) received the same story and the same list of idea units, but they received a picture booklet containing, in random order, the six detail and four relational pictures originally used by Waddill et al. (1988). They were given the same rating instructions as the first group. The purpose of this original picture group was to allow specific comparisons between the original pictures and the redrawn versions used in the present study.

Responses were scored as the proportion of targeted idea units correctly identified for each picture category (detail and relational). For detail pictures, subjects in the new picture group correctly identified an average of $84 \%$ of the targeted idea units $(S D=11 \%)$. Misidentifications were of two types. The subjects either identified a detail picture as depicting an idea unit we intended to target with a relational picture instead (i.e., a target relation) or they identified a detail picture as depicting idea units that we did not intend to target with either kind of picture (i.e., nontarget propositions). On average, the subjects identified $28 \%(S D=15 \%)$ of idea units targeted in relational pictures as also being depicted in the detail pictures and $37 \%(S D=17 \%)$ of the nontarget propositions as being depicted in the detail pictures.

For the relational pictures, subjects in the new picture group correctly identified an average of $47 \%(S D=17 \%)$ of the idea units we intended to target with relational pictures. For relational pictures, misidentifications consisted of identifying a relational picture either as depicting an idea unit targeted in a detail picture or as depicting a nontargeted idea unit. On average, the subjects identified $26 \%$ $(S D=11 \%)$ of the idea units targeted by detail pictures as also being depicted in relational pictures. They identified $44 \%$ of the nontarget idea units as also being depicted in the relational pictures.

These matching data (from the new picture group) were analyzed with a $2 \times 3$ within-subject analysis of variance (ANOVA) with picture type (detail, relational) and type of idea unit (target detail, target relation, nontarget) as factors. The results showed a significant main effect of idea unit $\left[F(2,20)=29.99, M S_{e}=.01\right]$ and a significant interaction of picture type with idea unit, $F(2,20)=$ 65.50, $\left.M S_{\mathrm{e}}=.01\right]$. Newman-Keuls analyses indicated that the subjects identified detail pictures as depicting a significantly higher proportion of target details than of target relations, and they identified relational pictures as depicting a significantly higher proportion of target relations than of target details. ${ }^{2}$

Upon closer inspection of the pattern of misidentified target information, we found that one relational picture contributed the most misidentifications. For this picture, 9 of the 11 subjects failed to identify a single one of the targeted relations but instead consistently identified a set of target details as being depicted by the picture. In effect, they were identifying this particular relational picture as being a detail picture. In subsidiary analyses reported in Experiment 2, we will consider the recall data of both Experiments 1 and 2 in light of this ineffective relational picture.

The matching results support our general contention that our detail and relational pictures were categorically different from one another. This is not to say that the pictures exclusively captured one type of information or the other. The pattern of misidentifications indicates that pictures that were intended to target details also captured some relational information (in the eyes of some subjects) and pictures intended to target relational information also reflected some details. What is important, however, is the relative weight accorded each kind of information in each kind of picture. The data confirm that our detail pictures were specifically representative of details (and of the details intended) and that they were processed as such. The specificity of the relational pictures for conveying the intended information was less; however, this is not surprising, because each relational picture targeted several concepts, and the subjects were less likely to identify all of the targeted ideas for each picture. Nevertheless, in general, the subjects did not appear to be interpreting the relational pictures as detail pictures, nor did they appear to be interpreting the detail pictures as relational pictures.

We also specifically compared identification accuracy for the subset of three redrawn detail and three redrawn relational pictures with their original versions from Waddill et al. (1988). Planned comparisons indicated that for detail pictures, subjects in the new picture group did not differ significantly from subjects in the original picture group in the proportion of correct identifications (84\% vs. $83 \% ; F<1$ ). For the relational pictures, however, significantly more target relations were identified in the redrawn pictures $(47 \%)$ than in the original versions of those pictures $(33 \%)\left[F(1,42)=4.85, M S_{e}=.01\right]$. This finding also supports the hypothesis that the new relational pictures were an improvement over those used in our earlier study.

Thus, it is possible that Waddill et al. (1988) failed to find mnemonic benefits for relational pictures because they used ineffective relational pictures. Our present findings also indicate that there are boundaries to Haring and Fry's (1979) conclusion that illustrations are generally ineffective in aiding the recall of details. The present work indicates that, at least with expository text and adult readers, the recall of details can be enhanced by illustrations. It appears that as long as pictures are designed to clearly depict detail or relational information, they will enhance the general recall of that information.

An important question, however, concerns the extent to which this straightforward pattern accurately reflects the impact of pictures on text memory for readers of dif- 
fering ability. With this question in mind and with the matching data corroborating our conceptual distinction between relational and detail pictures, we now turn to the presentation of Experiment 2.

\section{EXPERIMENT 2}

Reading comprehension may be defined as the extraction of meaning from a text and may be conceptualized by various processes, including decoding, accessing word meaning, and extracting relationships among idea units in a text (Golinkoff, 1976). For present purposes, we define comprehension skill in terms of the latter process. More skilled comprehenders are better at extracting relationships from a text and thus are better at constructing an effective memory representation of a passage than are less skilled comprehenders. In this experiment, we addressed the degree to which comprehension skill limits the mnemonic effects of pictures on recall performance. ${ }^{3}$

The general literature on individual differences in reading ability suggests four contrasting views of how such limitations might occur. To date, research into the relationship of pictures and reading ability has generally taken a compensatory stance. Theorists in this area have proposed a variety of compensatory functions for pictures within which we can outline two different frameworks. A general compensatory framework suggests that highly skilled comprehenders are already skilled at extracting and remembering information presented in texts, so representational aids such as pictures are superfluous. They add little to the memory representation that highly skilled comprehenders can construct from the text alone (see Mayer \& Gallini, 1990). The result is that illustrations will not benefit recall for these readers, regardless of the type of information the pictures convey. In contrast, less skilled comprehenders have difficulty constructing representations of textually presented information (Cooney \& Swanson, 1987) and need compensatory aids to help them adequately construct such representations (Mastropieri \& Scruggs, 1989; Mastropieri, Scruggs, \& Levin, 1987). Pictures might be expected to be especially useful for less skilled comprehenders, because pictures avoid the written format with which these learners have difficulty. The general compensatory view thus predicts that the mnemonic benefit of pictures for targeted information will be limited to (or, at least, especially pronounced for) less skilled comprehenders.

A selective compensatory framework offers a more complex variation on the theme that pictures serve to compensate for skills not naturally engaged by the reader. This view assumes that more skilled comprehenders are those who focus on the relational information in the text and attempt to form an organized, well-structured memory representation of the text (Levin, 1981). In doing so, however, they become less focused on particular details. In this view, relational pictures will be superfluous for highly skilled comprehenders, but detail pictures will not. The opposite would be the case for less skilled comprehenders.
These readers are not proficient at extracting the relational information in the text but focus on individual details instead (Golinkoff, 1976). Accordingly, for less skilled comprehenders detail pictures would be redundant with the kind of information that they are already encoding, but relational pictures would not. This framework thus predicts that relational pictures will primarily benefit less skilled comprehenders, and detail pictures will primarily benefit more highly skilled comprehenders.

Although intuitively appealing, the idea that pictures serve a compensatory role for reading deficits has not been supported by the limited research conducted so far (e.g., Harber, 1980; Rose, 1986). Readers may not benefit from even well-constructed pictures if those readers do not already possess the skills required for using pictures to construct memory representations (Larkin \& Simon, 1987). It may be that pictures incorporated into a text only "allow the rich to get richer" (see Salomon, 1972). In this general enrichment view, skilled comprehenders are able to exploit the pictures to construct even better and more entailed memory representations than they could construct from the text alone. Less able comprehenders, on the other hand, struggle to form a coherent representation regardless of whether it is presented in textual or pictorial format (Gernsbacher et al., 1990). This general enrichment framework predicts that pictures will enhance the recall of both detail and relational information for more highly skilled comprehenders but will have no benefit for less skilled comprehenders.

Finally, a selective enrichment approach is possible. Perhaps, as outlined in the selective compensatory view, good comprehenders focus more on relational processing than on detail processing, and less able comprehenders do the opposite (A. L. Brown \& Smiley, 1978; Golinkoff, 1976). If so, and if pictures serve to enrich the memory representation of information that already receives primary consideration, then relational pictures would enhance recall (of relational information) primarily for good comprehenders, but detail pictures would enhance recall (of detail information) primarily for less able comprehenders.

The primary motivation for the present experiment was to test the predictions of the four views just mentioned. We also had a secondary purpose. Another possible source of the difference between Waddill et al.'s (1988) original results and those of the present study may have been their using a less organized text. Perhaps the mnemonic benefits of embedded pictures depend upon the passage also containing explicit organizational cues in the surface structure of the text. In this experiment, therefore, we used a passage that was written with the same loose coherence among idea units and the same lack of overt relational signals as the passage originally used by Waddill et al. (1988). The top half of Table 3 presents an example of this version. We also used a second version of this same passage. This version was written with even more surface structure signals to the relationships among idea units than the version used in Experiment 1 (see bottom half 
Table 3

Experiment 2: An Example of Equivalent Information as Presented in the Unsignaled and Signaled Versions of the Experimental Text

\section{Unsignaled Version}

The hanging glaciers on Kanchenjunga are frequently of enormous thickness, with walls of ice 600 to 1,000 feet wide.

The hanging glaciers in the Alps rarely reach 200 feet wide.

Ice in the Alpine district is more brittle and breaks away more often.

There are more frequent avalanches in the Alps, but none approach the magnitude of Kanchenjunga avalanches.

\section{Signaled Version}

The plasticity of the ice allows Kanchenjunga to develop enormously thick hanging glaciers with walls of ice 600 to 1,000 feet wide.

By contrast, the hanging glaciers in the Alps rarely reach 200 feet wide, because the ice in the Alpine district is more brittle and breaks away more often.

Thus, although avalanches are more frequent in the Alps, none approach the magnitude of the Kanchenjunga avalanches.

of Table 3 for an example of this version). If the presence of explicit relational links in the text determines the efficacy of embedded relational illustrations, then the less explicit (unsignaled) version of the passage should show no benefit of relational illustrations on recall of relational information (replicating the results of Waddill et al.), but the more explicit (signaled) version should (replicating the results of Experiment 1).

\section{Method}

Subjects. The subjects were 180 Purdue University undergraduates who participated in partial fulfillment of an introductory psychology course requirement. The subjects were tested in groups of 1 to 4 people each.

Materials. Two versions of the passage from Experiment 1 were constructed. The two versions contained the same target information and were essentially paraphrases of one another. The primary difference between the two versions was that in one version (the signaled version), comparative and causal relationships among concepts were made even more explicit via connective words such as "by contrast," "more than," "because," and "thus." The unsignaled version was written in the same manner as the expository passage used by Waddill et al. (1988), which did not include such connectives. Table 3 provides an example of how identical target information was presented in the signaled and unsignaled versions. The unsignaled version consisted of 13 sentences and 62 idea units. The signaled version contained 18 sentences and 68 idea units.

Detail-picture, relational-picture, and no-picture control booklets were constructed for each version of the passage. The booklets were constructed in a manner similar to that used for Experiment 1, except that sentences containing unillustrated idea units were not placed on separate pages. Thus, each booklet consisted of four pages, and each page (except for the control condition) contained at least one picture illustrating information on that page. The pictures from Experiment 1 were used in this experiment as well.

Design and Procedure. The design was a 2 (passage version) $\times 3$ (picture type) $\times 3$ (skill level) between-subject factorial. The subjects were assigned to one of three comprehension skill levels on the basis of their scores on the comprehension subtest of the NelsonDenny Reading Test, Form D (J. I. Brown, Nelson, \& Denny, 1973). Any subject whose score did not fall within the limits for one of the three skill groups was excluded from subsequent analyses.

Subjects whose subtest scores fell at or below the 34th percentile (based on Nelson-Denny college norms) were designated less skilled comprehenders. This group consisted of 60 subjects with the average score of the group $(M=34)$ falling at the 19 th percentile. Subjects whose subtest scores fell between the 41 st and 69th percentiles were designated moderately skilled comprehenders. This group consisted of 60 subjects with the average score $(M=46)$ falling at the 54th percentile. Subjects whose subtest scores fell between the 74th and 99th percentiles were designated highly skilled comprehenders. This group consisted of 60 subjects with the average score $(M=56)$ falling at the 86th percentile.

The experimental procedure was identical to that used in Experiment 1 , except that the subjects took the Nelson-Denny comprehension subtest after they completed the postexperimental questionnaire. Eighty-one subjects indicated that they had expected and prepared for a memory test. These subjects were replaced with 81 naive subjects at the appropriate skill levels. Three other subjects who did not follow instructions were also replaced, as was one subject who had to be replaced due to an experimenter error.

\section{Results and Discussion}

Comprehension. Table 4 contains the means for reading time, comprehension rating, and rating of prior knowledge. There were no significant differences in prior knowledge ratings (all $F \mathrm{~s}<1$ ), with ratings uniformly reflecting low prior knowledge of the passage content. For the comprehension ratings, there was no main effect of passage version $(F<1)$ or skill level $[F(2,162)=$ $\left.1.82, M S_{\mathrm{e}}=.60\right]$. There was a significant main effect for picture type $\left[F(2,162)=3.47, M S_{\mathrm{e}}=.60\right]$, which was modified by a significant interaction with skill level $\left[F(4,162)=3.27, M S_{\mathrm{e}}=.60\right]$. A Newman-Keuls analysis indicated highly skilled comprehenders rated a passage accompanied by relational pictures as significantly more comprehensible than one accompanied by detail pictures. Detail pictures did not significantly affect their comprehension ratings, relative to no pictures. Moderately skilled comprehenders rated a passage accompanied by relational pictures as more comprehensible than both a passage accompanied by detail pictures and a passage without pictures. The addition of detail pictures did not

Table 4

Experiment 2: Mean Reading Times, Comprehension Ratings, and Prior Knowledge Ratings as a Function of Picture Condition and Comprehension Skill Level

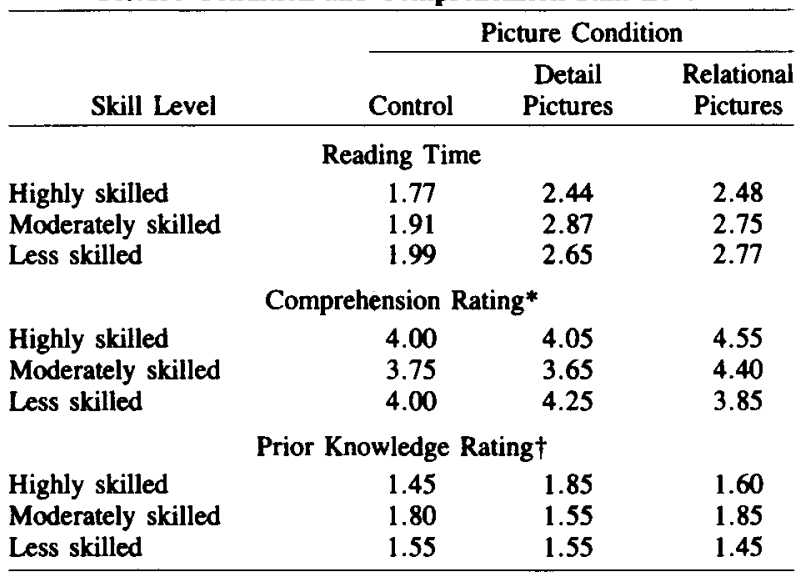

Note-Reading time is given in minutes. * Larger numbers indicate higher comprehension rating (highest possible $=5$ ). †Larger numbers indicate higher prior knowledge rating (highest possible $=7$ ). 
significantly affect their comprehension ratings, relative to no pictures. For less skilled comprehenders, pictures had no effect on comprehension ratings.

Reading time differed significantly as a function of skill level $\left[F(2,162)=5.03, M S_{e}=.28\right]$. A Newman-Keuls analysis revealed that highly skilled comprehenders read faster than moderately skilled and less skilled comprehenders (whose reading times did not significantly differ from each other). There was also a significant main effect of passage version $\left[F(1,162)=4.32, M S_{\mathrm{e}}=.28\right]$. Reading time was longer for the signaled version $[M=$ $2.48 \mathrm{~min})$ than for the unsignaled version $(M=2.32 \mathrm{~min})$. The only other significant effect was a main effect of picture type $\left[F(2,162)=43.01, M S_{\mathrm{e}}=.28\right]$. A NewmanKeuls analysis indicated that the no-picture version was read faster $(M=1.89 \mathrm{~min})$ than either the detail-picture version or the relational-picture version, which did not differ from each other $(M=2.65$ and $2.67 \mathrm{~min}$, respectively).

Free recall. Two scorers scored a random subset of 10 protocols using the same rating method described in Experiment 1. The subset consisted of two protocols from the high-skill control group and one protocol from each of the other nine skill $\times$ version groups. Scorers were blind to the condition from which each protocol was drawn. Because the correlation between the raters was .93, the remaining protocols were scored by one of the scorers.

First, we evaluated the reliability of our findings in Experiment 1 via planned comparisons collapsed over reading ability. Because there was no main effect of passage version and no interaction of passage version with any other factor (all $F \mathrm{~s}<1$ ), these and all subsequent analyses were also collapsed across passage version. As in Experiment 1, the error terms in this and subsequent analyses were taken from separate analyses of each type of recall (target detail, target relational, nontarget). The planned comparisons confirmed the reliability of the results obtained in Experiment 1. Recall of targeted relational information was significantly greater in the relational picture condition than in the no-picture condition $\left[F(1,114)=28.24, M S_{e}=.01\right]$ but detail recall was not significantly enhanced by relational pictures $[F(1,114)=$ $\left.2.69, M S_{e}=.03\right]$. Similarly, recall of targeted detail information was significantly greater in the detail picture condition than in the no-picture condition $[F(1,114)=$ $31.17, M S_{\mathrm{e}}=.03$ ], but recall of relational information was not significantly enhanced $\left[F(1,114)=2.81, M S_{\mathrm{e}}=\right.$ $.01]$. Recall of nontargeted information was not affected by the addition of either picture type (all $F s<1$ ).

To assess how comprehension skill per se related to text recall, we first performed separate one-way ANOVAs (with reading ability as the independent variable) for recall of each type of information for those subjects in the no-picture control group. There was no significant effect of comprehension skill in any of these analyses (all $F$ s $<1$ ).

Next, we evaluated the theoretical predictions of the four views outlined in the introduction to Experiment 2. Because we were testing specific a priori predictions, we analyzed the data with the more appropriate planned comparison procedure rather than with an omnibus ANOVA and post hoc tests (see Rosenthal \& Rosnow, 1985, for justification of this statistical approach). The critical comparisons were between the control (no-picture) group and each of the picture groups within each skill level. Table 5 presents the mean proportion of the total possible scores for target and nontarget propositions at each skill level.

The general mnemonic benefit for relational pictures observed in Experiment 1 was found to be limited to certain reading levels. Relational pictures significantly enhanced the recall of target relational information (relative to the no-picture control) for highly skilled and moderately skilled comprehenders $[F(1,114)=15.67$ and $F(1,114)=13.49$, respectively; $\left.M S_{\mathrm{e}}=.01\right]$, but not for less skilled comprehenders $\left[F(1,114)=2.48, M S_{e}=\right.$ $.01]$. Moreover, for highly skilled comprehenders, the mnemonic benefit of relational pictures extended to recall of target details. That is, for highly skilled comprehenders only, specific details (that were encompassed by, but not explicitly depicted in, the relational pictures) were better recalled in the relational picture condition than in the no-picture control $\left[F(1,114)=5.61, M S_{\mathrm{e}}=.03\right]$. Recall of nontarget information was not enhanced by relational pictures for any comprehension skill level (largest $\left.F=1.29, M S_{\mathrm{e}}=.004\right)$.

The mnemonic benefit of detail pictures also appeared to be modulated somewhat by comprehension skill level: less skilled and moderately skilled comprehenders appeared to benefit more in recall of details than did highly skilled comprehenders. Comparisons of the detail picture conditions with the no-picture conditions at each skill level,

Table 5

Experiment 2: Mean Proportion of Total Possible Score for Free Recall of Target Detail, Target Relational, and Nontarget Information as a Function of Comprehension Skill Level and Picture Condition (Collapsed Across Passage Version)

\begin{tabular}{llll}
\hline & \multicolumn{3}{c}{ Picture Condition } \\
\cline { 2 - 4 } $\begin{array}{c}\text { Skill Level and } \\
\text { Information Type }\end{array}$ & Control & $\begin{array}{c}\text { Detail } \\
\text { Pictures }\end{array}$ & $\begin{array}{c}\text { Relational } \\
\text { Pictures }\end{array}$ \\
\hline & Highly Skilled & \\
Target detail & $.22(.22)$ & $.35(.33)$ & $.35(.25)$ \\
Target relational & $.16(.17)$ & $.19(.18)$ & $.26(.27)$ \\
Nontarget & .12 & .13 & .11 \\
& Moderately Skilled & \\
Target detail & $.20(.21)$ & $.40(.34)$ & $.27(.21)$ \\
Target relational & $.13(.13)$ & $.15(.13)$ & $.22(.23)$ \\
Nontarget & .08 & .11 & .10 \\
& Less Skilled & $.42(.38)$ & $.16(.10)$ \\
Target detail & $.20(.19)$ & $.16(.15)$ & $.17(.17)$ \\
Target relational & $.13(.15)$ & .09 & .07 \\
Nontarget & .10 & & \\
\hline
\end{tabular}

Note-Scores reflect the proportions of the total possible scores for target detail, target relational, and nontarget information collapsed across unsignaled text version (24, 40, and 60, respectively) and signaled text version $(24,40$, and 72 , respectively). Scores in parentheses represent total scores after elimination of problematic relational picture from analysis (see text for explanation). 
however, showed that detail pictures significantly enhanced the recall of target details for all three groups [less skilled comprehenders, $F(1,114)=14.56, M S_{\mathrm{e}}=.03$; moderately skilled comprehenders, $F(1,114)=12.65, M S_{\mathrm{e}}=$ .03 ; highly skilled comprehenders, $F(1,114)=5.28$, $M S_{\mathrm{e}}=.03$ ]. Recall of relational information was not increased by detail pictures for any skill level (largest $F=$ $1.47, M S_{\mathrm{e}}=.01$ ), nor was recall of nontarget information enhanced by detail pictures at any skill level (largest $F=1.52, M S_{\mathrm{e}}=.01$ ).

Subsidiary analyses. As indicated in the picturematching results, one of our relational pictures was consistently treated as a detail picture. In fact, raters consistently associated it with a set of details also targeted by one of our detail pictures. It is possible, therefore, that this misdrawn picture elicited the processing of details more than it did the processing of the relations we intended it to target. If this were the case, then this picture might have artificially inflated the mean recall of details by the relational picture groups. We therefore reanalyzed the data from Experiments 1 and 2, eliminating from the analyses both the intentionally targeted relations and the details apparently targeted by the misdrawn relational picture. The pattern of results in Experiment 1 remained unchanged (Table 2 includes the adjusted means compared in this reanalysis). When the data from Experiment 2 were reanalyzed, the pattern of results showed an interesting change (Table 5 includes the adjusted means used in these analyses). Comparisons of these new, adjusted means with the adjusted means for the respective control groups indicated, as expected, no change from the initial analysis of detail-picture effects. Detail pictures produced significantly greater recall of target details (relative to no pictures) for all skill levels. Relational pictures, however, no longer showed an enhancement of target-detail recall for highly skilled comprehenders $(F<1)$. For less skilled comprehenders, the addition of relational pictures produced a marginally significant decrease in recall of target details $\left[F(1,114)=2.70, p<.06, M S_{\mathrm{e}}=.03\right]$.

\section{GENERAL DISCUSSION}

In the present research, we asked two questions. The first was whether or not illustrations could be devised that would enhance the processing of pictured information in an expository text regardless of the type of information depicted (relational or detail). Previous work with illustrations and expository text had failed to find beneficial effects of pictures for recall of relational information (Waddill et al., 1988). Furthermore, other researchers (Haring \& Fry, 1979) contended that pictures in general were an ineffective method of enhancing the recall of details from text. The results of Experiment 1, including the picture-matching data, indicate that pictures can indeed enhance the recall of both relational and detail information from expository text, at least with college students.
The present work also suggests that Waddill et al.'s (1988) failure to find effects of relational pictures with expository text may have been due more to the nature of their relational pictures than to the organizational structure of their passage and suggests a reinterpretation of their general conclusion that without special processing instructions, pictures will not enhance relational processing in expository passages. In Experiment 2, we used two versions of the expository passage, one with relatively loose conceptual coherence and little explicit signaling of relational links among idea units (such as that used by Waddill et al.) and one in which the conceptual relationships were made even more explicit than those in the passage from Experiment 1. If the effects of relational illustrations were dependent upon the degree to which relational links are signaled in a text, then relational pictures should have produced no effects for the unsignaled version (paralleling Waddill et al.'s original findings) but should have enhanced relational recall in the signaled version (paralleling the results for the passage used in Experiment 1). Our data indicated no such pattern. Both the unsignaled and signaled versions produced the same general effects found in Experiment 1: Detail pictures enhanced recall of target details, relational pictures enhanced recall of target relations, and recall of nontarget information was not affected by either kind of picture.

The primary issue motivating Experiment 2 was the possibility that the effects of pictures on memory for detail and relational information were affected by individual differences in comprehension skill. The recall patterns in Experiment 2 were inconsistent both with the general assumption that embedded pictures serve a primarily compensatory role for less able readers (Levin, 1981; Levin, 1983) and with a more selective compensatory view. As outlined earlier, these frameworks assume that encoding manipulations, such as embedded pictures, will be effective to the extent that they encourage readers to focus on the kinds of information they would not, in the absence of such manipulations, adequately encode into their memory representation. As Experiment 2 indicated, illustrations embedded in a text enhanced recall (relative to an unillustrated text) for highly skilled and moderately skilled comprehenders. Less skilled comprehenders benefited only very selectively from embedded illustrations, with the benefit restricted to recall of targeted details. The finding that less skilled comprehenders benefited from pictures, however selectively, also contradicted the general enrichment hypothesis, which assumed that only the more able comprehenders would profit from pictures.

A selective enrichment view, which suggests that pictures should serve an enriching function for the kind of information that already receives primary consideration by the reader, is more consistent with the present results. Highly skilled comprehenders are adept at encoding relational information and building coherent textual representations (Mayer \& Gallini, 1990), and adding relational pictures to the text bolstered these processes and increased 
recall of relational information. The less skilled comprehenders apparently were able to build representations of simple details from a passage, and adding detail pictures enhanced their processing and recall of such information. They seemed, however, to be inefficient at building coherent memory representations during comprehension (this inefficiency in encoding relational information is general across written, spoken, and pictorial input; see Gernsbacher et al., 1990). Pictures designed to depict relationships did not help these less skilled comprehenders recall relational information. In fact, for these readers, relational pictures actually produced a marginally significant $d e-$ crease in the recall of target details, relative to an unillustrated text.

There are several findings, however, that suggest some necessary refinements to the selective enrichment framework. An assumption of the selective enrichment view is that highly skilled comprehenders focus more on relational information, whereas less skilled comprehenders focus more on details. In the present study, the absence of differences in recall of detail and relational information among different skill-level groups in the no-picture control condition questions this idea. A selective enrichment view also predicts that detail pictures should not enhance highly skilled comprehenders' recall of target details, because these readers tend to concentrate on relational information and may disregard specific details. The data from the present study also fail to support this prediction. Detail pictures enhanced recall of targeted details for highly skilled comprehenders as well as for less skilled comprehenders.

One way to view the entire pattern of results is that pictures enable the extraction and retention of information that readers, under ordinary circumstances, do not encode well enough to recall, even though they may have the requisite abilities to do so (see Salomon, 1974, 1979). More skilled readers may not have concentrated on all of the information in a passage any more efficiently than less skilled readers did under the current task demands, but the more skilled group was capable of activating the appropriate encoding processes if it seemed important to do so. Indeed, one hallmark of good comprehenders seems to be their ability to pay attention to information relevant to their purpose and ignore information that is not useful for the task (Golinkoff, 1976). That is, good readers seem sensitive to the general importance of information in a text, but they are also sensitive to signals indicating the relevance of information to subsequent tasks (Schraw \& Wade, 1991; Weidenmann, 1989). Thus, good readers will attend to and remember information that they do not ordinarily consider important if that information is signaled as being relevant. In the present context, the detail pictures may have served as such a signal for the targeted detail information.

Less skilled readers possess the skills needed to effectively encode details into their memory representations, and these skills are even more efficiently engaged with pictures. Because of the limitations faced by these readers in terms of encoding relational information (see Gernsbacher et al., 1990), enablement through relational pictures is apparently not possible because the requisite skills are not there to begin with (Larkin \& Simon, 1987).

When considering how individual differences modulate the effects of pictures on prose retention, it should be noted that readers may experience reading difficulty for a variety of reasons, including sensory impairment, learning disabilities, inadequate decoding skills, emotional interference (Golinkoff, 1976), and low working-memory capacity (Just \& Carpenter, 1992; Lee-Sammons \& Whitney, 1991). The present patterns may not hold for all of these different kinds of poor comprehenders. Furthermore, evidence indicates that developmental differences exist in readers' ability to efficiently employ various textbased adjuncts and encoding manipulations (Ackerman, 1986; Hartley \& Trueman, 1985), and such differences may exist for pictures as well (see Schallert, 1980). Our subjects were all college students. Haring and Fry's (1979) subjects were children rather than adults, which may have accounted for their failure to find pictorial enhancement of detail recall. Nevertheless, it is important to note that even though we were dealing with a range of ability among competent college readers, the mnemonic effects of pictures were quite robust, and those effects varied according to different levels of comprehension skill.

Two other factors that may influence the effects of pictures on recall are task demands (e.g., reading objectives) and individual differences in prior knowledge. We used an incidental learning situation; however, under intentional learning instructions, the present patterns might change. ${ }^{4}$ We also used subjects with low prior knowledge of our passage information (as indicated by their selfratings). Mayer and Gallini (1990) found that pictures could serve a compensatory function when those pictures were designed to help readers build mental models of a passage and the readers had little prior knowledge of the passage topic. Our subjects' self-ratings of their prior knowledge of content of the expository passage showed that they knew very little about the topic. Our results extend the findings of Mayer and Gallini by showing that within a group of low-knowledge readers, even further differentiation is possible on the basis of individual differences in comprehension skill. In such cases, pictures seem to enable readers to extract and remember information that they might not otherwise encode well enough to recall, provided that they already possess the requisite encoding skills.

\section{REFERENCES}

ACKerman, B. P. (1986). The use of item-specific and relational episodic information in the recall of children and adults. Journal of Experimental Child Psychology, 42, 115-143.

Bransford, J. D., Johnson, M. K. (1972). Contextual prerequisites for understanding. Joumal of Verbal Leaming \& Verbal Behavior, 11, 717-726.

Brown, A. L., SMILEY, S. S. (1978). The development of strategies for studying texts. Child Development, 49, 1076-1088. 
Brown, J. I., Nelson, M. J., \& Denny, E. C. (1973). The NelsonDenny Reading Test, Form D. Boston: Houghton-Mifflin.

CoOney, J. B., Swanson, H. L. (1987). Memory and learning disabilities: An overview. In H. L. Swanson (Ed.), Memory and learning disabilities: Advances in learning and behavioral disabilities ( $\mathrm{pp} .1$ 40). Greenwich, CT: JAI.

Einstein, G. O., McDaniel, M. A., Bowers, C. A., Stevens, D. T. (1984). Memory for prose: The influence of relational and proposition-specific processing. Journal of Experimental Psychology. Learning, Memory, \& Cognition, 10, 133-143.

Einstein, G. O., McDaniel, M. A., Owen, P. D., \& Cote, N. C. (1990). Encoding and recall of texts: The importance of material appropriate processing. Journal of Memory and Language, 29, 566-581.

FELDMAN, M. J. (1985). Evaluating pre-primer basal readers using story grammar. American Educational Research Journal, 22, 527-547.

Gernsbacher, M. A., Varner, K. R., FAust, M. (1990). Investigating differences in general comprehension skill. Journal of $E_{x}$ perimental Psychology: Learning, Memory, \& Cognition, 16, 430-445.

GolinkofF, R. M. (1976). A comparison of reading comprehension processes in good and poor comprehenders. Reading Research Quarterly, 11, 623-659.

HARBER, J. R. (1980). Effects of illustrations on reading performance: Implications for further LD research. Learning Disability Quarterly, 3, 60-70.

Haring, M. J., \& FrY, M. A. (1979). Effect of pictures on children's comprehension of written text. Educational Communication \& Technology, 27, 185-190.

Hartley, J., \& Trueman, M. (1985). A research strategy for text designers: The role of headings. Instructional Science, 14, 99-155.

Just, M. A., \& CARPENTER, P. A. (1992). A capacity theory of comprehension: Individual differences in working memory. Psychological Review, 99, 122-149.

KINTSCH, W. (1974). The representation of meaning in memory. Hillsdale, NJ: Erlbaum.

LARKIN, J. H., \& SIMON, H. A. (1987). Why a diagram is (sometimes) worth ten thousand words. Cognitive Science, 11, 65-99.

LeE-Sammons, W. H., WhitneY, P. (1991). Reading perspectives and memory for text: An individual difference analysis. Journal of Experimental Psychology: Leaming, Memory, \& Cognition, 17, 1074-1081.

LEVIN, J. R. (1973). Inducing comprehension in poor readers: A test of a recent model. Journal of Educational Psychology, 65, 19-24.

LEVIN, J. R. (1981). On functions of pictures in prose. In F. J. Pirozzolo \& M. C. Witrock (Eds.), Neuropsychological and cognitive processes in reading (pp. 203-228). New York: Academic Press.

Levin, J. R. (1983). Pictorial strategies for school learning: Practical illustrations. In M. Pressley \& J. R. Levin (Eds.), Cognitive strategy research: Educational applications (pp. 213-237). New York: SpringerVerlag.

Levin, J. R., Anglin, G. J., \& Carney, R. N. (1987). On empirically validating functions of pictures in prose. In D. M. Willows \& H. A. Houghton (Eds.), The psychology of illustration: Vol. 1. Basic research (pp. 51-85). New York: Springer-Verlag.

Mastropieri, M. A., Scruggs, T. E. (1989). Constructing more meaningful relationships: Mnemonic instruction for special populations. Educational Psychology Review, 1, 83-111.

Mastropier,, M. A., SCrugGs, T. E., \& LeVIN, J. R. (1986). Leaming disabled students' memory for expository prose: Mnemonic vs. nonmnemonic pictures. American Educational Research Joumal, 24, 505-519.
MaYer, R. E., \& Gallini, J. K. (1990). When is an illustration worth ten thousand words? Joumal of Educational Psychology, 82, 715-726. McDaniel, M. A., Einstein, G. O., Dunay, P. K., \& Cobb, R. E. (1986). Encoding difficulty and memory: Toward a unifying theory. Journal of Memory \& Language, 25, 645-656.

ORTONY, A. (1978). Remembering, understanding, and representation. Cognitive Science, 2, 53-69.

Rose, T. L. (1986). Effects of illustrations on reading comprehension of learning disabled students. Journal of Learning Disabilities, 19 , 542-544.

Rosenthal, R., \& Rosnow, R. (1985). Contrast analysis: Focused comparisons in the analysis of variance. Cambridge, MA: Cambridge University Press.

SALOMON, G. (1972). Heuristic models for the generation of aptitudetreatment interaction hypotheses. Review of Educational Research, 42, 327-343

SALOMON, G. (1974). Internalization of filmic schematic operations in interaction with learners' aptitudes. Journal of Educational Psychology, 66, 499-511.

SALOMON, G. (1979). Media and symbol systems as related to cognition and learning. Journal of Educational Psychology, 71, 131-148.

SCHALLERT, D. L. (1980). The role of illustrations in reading comprehension. In R. J. Spiro, B. C. Bruce, \& W. F. Brewer (Eds.), Theoretical issues in reading comprehension (pp. 503-524). Hillsdale, $\mathrm{NJ}$ : Erlbaum.

Schraw, G., \& WADE, S. (1991, April). Selective learning strategies for relevant and important text information. Paper presented at the Annual Meeting of the American Educational Research Association, Chicago.

Waddill, P. J., McDaniel, M. A., \& Einstein, G. O. (1988). Illustrations as adjuncts to prose: $\mathrm{A}$ text-appropriate processing approach Journal of Educational Psychology, 80, 457-464.

WeidenMANN, B. (1989). When good pictures fail: An informationprocessing approach to the effect of illustrations. In $\mathrm{H}$. Mandl \& J. R. Levin (Eds.), Knowledge acquisition from text and pictures (pp. 157 170). Amsterdam: Elsevier, North-Holland.

\section{NOTES}

1. We thank an anonymous reviewer for suggesting this possibility.

2. Our primary interest was a functional one: How well do different types of pictures (detail or relational) produce a focus on the corresponding type of information (i.e., detail or relational)? For present purposes, therefore, we were not specifically concerned with how accurate subjects were in assigning to each picture the particular idea units we in tended to target with that picture. We did, however, calculate for each subject the average proportion of identified idea units not associated with the "right" picture. The subjects assigned an average of $9 \%$ ( $S D=$ $13 \%$ ) of the targeted details to the "wrong" detail picture and assigned an average of $40 \%$ ( $S D=17 \%)$ of the targeted relations to the "wrong" relational picture.

3. We do not intend to imply that comprehension and recall are identical. We are simply acknowledging that comprehension relates to the construction of memory representations, which can, in turn, influence recall performance (Bransford \& Johnson, 1972; Ortony, 1978).

4 . We thank the reviewers for raising this point.

(Manuscript received August 14, 1991; revision accepted for publication March 11, 1992.) 